\title{
Displasia evolutiva de cadera: controversia sobre el cribado ecográfico universal
}

\author{
M. Paz Lovera, L. Llorente Otones, I. Rivero Calle, \\ L. Lesmes Moltó, MR. Rodríguez Díaz, MJ. Rivero Martín \\ Servicio de Pediatría. Hospital de Fuenlabrada. Fuenlabrada, Madrid.
}

\begin{abstract}
Resumen
Pese a la elevada incidencia de displasia de cadera clínicamente importante (3-5 casos por cada 1000 recién nacidos vivos) y a los ampliamente estudiados factores de riesgo principales (parto de presentación podálica, sexo femenino y antecedentes familiares de displasia evolutiva de cadera), no se ha conseguido encontrar una estrategia efectiva que reduzca la incidencia de casos tardíos. Reabrimos, a propósito de un caso, la controversia existente acerca del cribado ecográfico universal en la patología osteomuscular más frecuente de la infancia.
\end{abstract}

Palabras clave: Displasia de cadera. Cribado. Ecografía.

Developmental hip dysplasia: controversy on universal echographic screening

Abstract

Despite the elevated incidence of clinically relevant hip dysplasia (3-5 per 1000 live births) and the broadly studied main risk factors (breech position, female sex and family cases), we have not achieved an effective prevention strategy, and so the detection of late cases has not decreased. We reopen, following a case, the existent controversy upon universal ultrasound screening on the most frequent musculo-skeletal disorder in the infancy.

Key words: Hip dysplasia. Screening. Ecography.

\section{Introducción}

La displasia evolutiva de cadera es la patología osteomuscular más frecuente en niños menores de dos años'. Las consecuencias de la detección tardía pueden ser significativas y se cuenta con los medios para diagnosticarla precozmente; sin embargo, sigue habiendo niños con diagnósticos tardíos $y$, por ende, con peores resultados funcionales ${ }^{2}$. El caso que presentamos nos ayuda a tener una perspectiva diferen-

Manuel Paz Lovera,doc_paz@yahoo.com

Los autores declaran no presentar conflictos de intereses en relación con la preparación y publicación de este artículo. 
te en el aún no resuelto debate sobre la necesidad y conveniencia de una política de cribado universal de esta patología.

\section{Caso clínico}

Niña de 18 meses, sin antecedentes personales ni familiares de interés, cuya madre refiere que desde que comenzó a caminar, hace apenas unos días, lo hace con una visible cojera.

La exploración revelaba una niña de aspecto saludable, bien nutrida, sonriente. Los hallazgos anormales se limitaban al examen ortopédico: asimetría de pliegues inguinales y glúteos, acortamiento del miembro inferior izquierdo de 1,5 $\mathrm{cm}$, discreta limitación a la abducción de cadera izquierda, no dolorosa, e hiperlordosis lumbar. A su vez, destacó un patrón de marcha anormal, oscilante, "de pato", de Trendelemburg. La radiografía de pelvis mostró un acetábulo displásico y cadera izquierda luxada (figuras 1 y 2 ).

\section{Comentario}

Displasia evolutiva de cadera (DEC) es el término utilizado actualmente para designar una condición en la que la cabeza femoral tiene una relación anormal con el acetábulo. Comprende la luxación (completa o parcial), la inestabili- dad o la luxabilidad, y también cambios radiológicos que reflejan una formación inadecuada del acetábulo. Se prefiere "evolutiva" a congénita porque los cambios no siempre son detectables al nacimiento, y "displasia" en vez de luxación porque se amplía el espectro de cambios que es posible encontrar en estos pacientes.

La incidencia de DEC varía según los medios de detección, la edad del paciente y los criterios diagnósticos ${ }^{4}$. No existe un patrón oro definitivo. Aun así, se puede decir que se trata de la anomalía musculoesquelética más frecuente de la infancia. Se acepta de manera general que la presencia de inestabilidad o displasia de cadera clínicamente importantes afecta a 3-5 de cada 1000 nacidos vivos ${ }^{5}$.

La patogénesis es multifactorial: factores mecánicos (presentación podálica, oligohidramnios, primogénitos, etc); laxitud ligamentosa (inducida por las hormonas maternas que relajan el suelo pélvico); herencia ${ }^{6,7}$. Aunque se ha descrito la displasia acetabular primaria, actualmente se acepta que la displasia es consecuencia de la relación anormal entre el fémur y el acetábulo, y no su causå.

Se han descrito varios factores de riesgo (FR). Los más importantes desde el punto de vista de los estudios y recomendaciones de cribado son: 1) presen- 
Figura 1. Displasia de cadera izquierda (proyección anteroposterior).

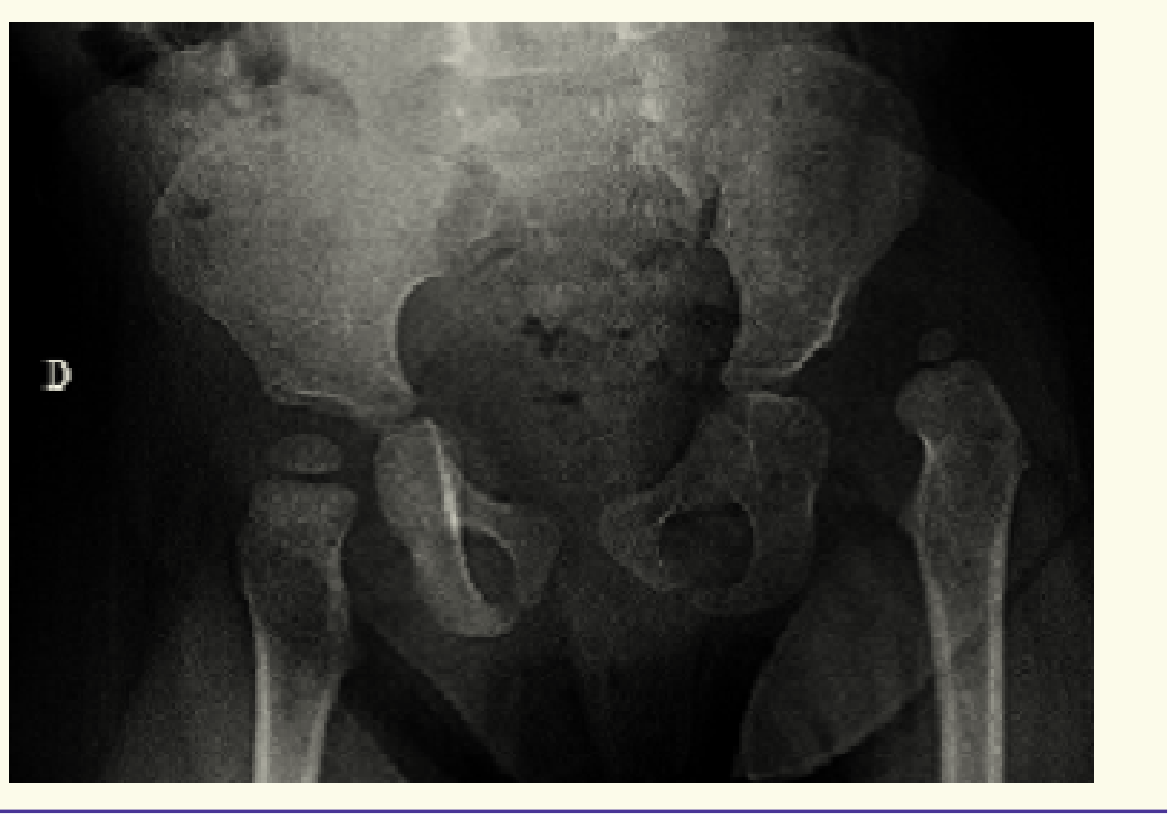

Figura 2. Displasia de cadera izquierda (proyección axial).

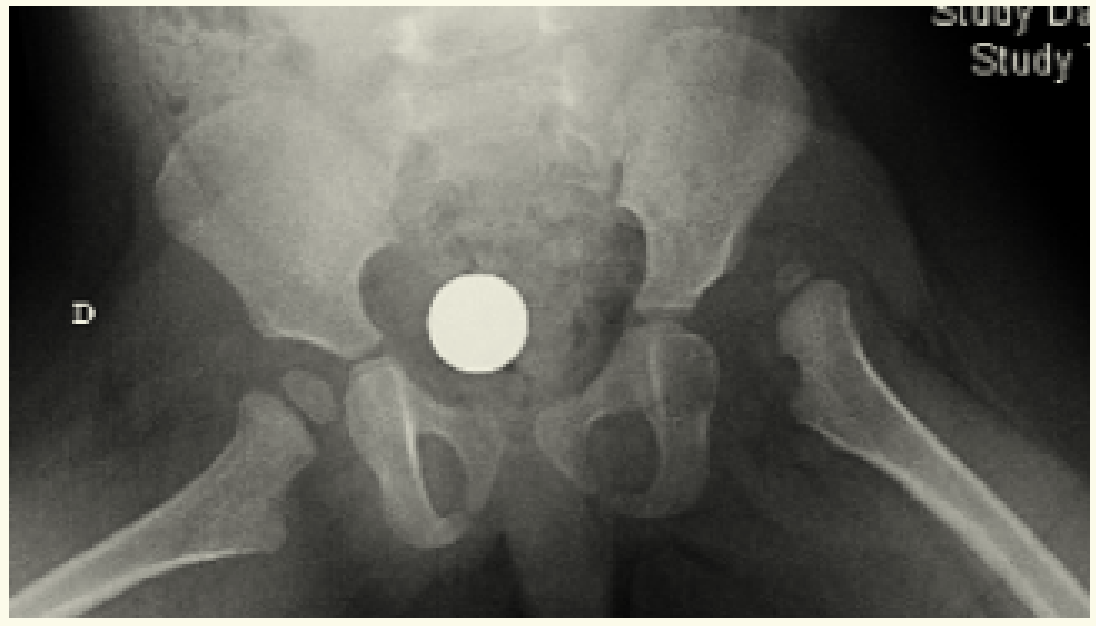


tación podálica; 2) sexo femenino, y 3) antecedentes familiares de DEC. También se ha descrito asociación con otras anomalías musculoesqueléticas: tortícolis congénito, metatarsus adductus, talipes equinovarus o calcaneovalgus, primogénito, entre otros 6 . Si bien es cierto que en niños que presentan estos FR la DEC es más frecuente, la realidad es que la mayoría de los pacientes con DEC no los presentan. Solo el 10-27\% de los pacientes con DEC tienen $\mathrm{FR}^{6,9}$. Es decir, el típico paciente con DEC no presenta ninguno de estos factores de riesgo, salvo el sexo femenino ( $80 \%$ ).

El tratamiento de la DEC es más sencillo y exitoso si se comienza de manera precoz (antes de los seis meses). Aunque la exploración de la cadera es la maniobra de evaluación musculoesquelética más frecuente el primer año de vi$\mathrm{da}^{10}$, la prevalencia de casos tardíos se ha mantenido estable ${ }^{11}$. A primera vista, y dados los recursos disponibles para el clínico actualmente, esto nos resulta cuanto menos desconcertante, pero una revisión de la bibliografía reciente parece inclinar ostensiblemente la balanza hacia una política de cribado clínico universal y uso de la ecografía de forma selectiva ${ }^{12-14}$.

Existe evidencia a favor del cribado universal. Zenios et al. estudiaron el im- pacto de una política de cribado selectivo durante cinco años: no redujo la incidencia de casos de presentación tardía ${ }^{15}$. Existe también evidencia en contra. Dos de los consensos más importantes y extensos publicados no lo recomiendan. El primero, de la Asociación Americana de Pediatría, en el año 2000, recomienda ecografía a las seis semanas de vida solo en niñas con antecedentes familiares de displasia y en todos los nacidos en presentación podálica ${ }^{12}$. En Canadá, otra extensa revisión publicada en el año 2001 contempla la exploración clínica únicamente durante el primer año de vida en todos los niños y ecografía solo ante sospecha clínica, obviando realizarla en lactantes con $\mathrm{FR}^{13}$. La complejidad de los datos se refleja mejor aún en otra revisión, esta vez de los servicios preventivos estadounidenses. Estos concluyen que, aunque el cribado ecográfico universal permite detectar más tempranamente la DEC, la alta tasa de resolución espontánea y la mala calidad de la evidencia hacía imposible realizar recomendaciones definitivas a favor o en contra ${ }^{14}$. Mencionaremos también, por el importante tamaño de la muestra, un estudio prospectivo publicado en 2002 que incluyó 28676 niños, de los que se aplicó cribado ecográfico selectivo a aquellos con FR o sospecha clínica: los resultados obteni- 
dos pusieron de manifiesto la incapacidad del uso de la ecografía selectiva para disminuir la necesidad de cirugía con respecto a la exploración física aislada ${ }^{15}$.

Si se aplicase una política de cribado ecográfico universal en el periodo neonatal, y se tratase a todos aquellos con una cadera con alteraciones, no cabe duda de que se estaría sometiendo a un tratamiento innecesario a la mayoría de ellos, dado que la evolución natural de la mayor parte de estas alteraciones es hacia la resolución espontánea ${ }^{16-20}$. Las desventajas del tratamiento innecesario son múltiples; entre ellas, la necrosis avascular de la cabeza del fémur. Sin embargo, la frecuencia de esta complicación es directamente proporcional al tiempo que se tarde en instaurar el tratamiento. En los casos de displasia leve, la frecuencia de esta complicación está entre 0 y $1 \%{ }^{21}$. En la inmensa mayoría son necrosis de grado I, siguen una evolución espontánea y no dejan secuelas ${ }^{22}$. No olvidemos tampoco los efectos psicológicos sobre los padres ${ }^{23}$ y las dificultades en la interacción madre-hijo en niños en tratamiento con arnés ${ }^{24}$. Además, el carácter evolutivo de la DEC determinará que niños con un cribado ecográfico negativo inicial desarrollen más adelante la enfermedad, por lo que ni aun así se evitan por completo los ca- sos de presentación tardía subsidiarios de cirugía.

En algún punto entre estas dos propuestas tiene que existir la mejor solución, una que no consienta que sigan apareciendo casos que requieran tratamiento quirúrgico y con alto riesgo de secuelas funcionales, y que a la vez permita que los casos que van a evolucionar espontáneamente a la curación lo hagan sin que se sometan a un tratamiento innecesario no exento de complicaciones y efectos secundarios.

El seguimiento cuidadoso por profesionales experimentados en el diagnóstico y manejo de la DEC de los lactantes que en un primer estudio (hecho antes de las seis semanas) tengan signos ecográficos de displasia leve sería una opción que hasta ahora ya ha demostrado ser una estrategia segura en países como Alemania, Reino Unido y Eslovenia, dejando evolucionar a la curación espontánea a la mayoría y, a la vez, captando a ese pequeño grupo de los que se beneficiarán de un tratamiento precoz y que por otros medios (antecedentes y examen físico) pasarían inadvertidos. Esta estrategia ha demostrado a su vez disminuir la necesidad de resolución quirúrgica sin incrementar los costes sanitarios ${ }^{25-27}$.

No pretendemos dar una respuesta definitiva para un problema sobre el 
que existe tanta controversia y sobre el que se han pronunciado profesionales con gran experiencia. El cribado universal puede llevar a sobrediagnóstico y sobretratamiento, pero no necesariamente de forma ineludible. Una primera ecografía positiva podría indicar solo la necesidad de un seguimiento individualizado. Por otro lado, está demostrado que el cribado selectivo deja de detectar, aun en las mejores manos, a un contingente de niños que acaban requiriendo tratamientos más agresivos y con peor pronóstico funcional. No parece que exista una estrategia perfecta, pero nos cuesta trabajo conformarnos con la idea de que en la España de este siglo sigamos viendo niños con diagnóstico tardío y alto riesgo de padecer secuelas de por vida a causa de una patología fácil de prevenir. Podemos y debemos hacerlo mejor.

\section{Bibliografía recomendada}

1. Aronsson DD, Goldberg MJ, Kling TF Jr, Roy DR. Developmental dysplasia of the hip. Pediatrics. 1994:94:201-8.

2. Burger BJ, Burger JD, Bos CF, Obermann WR, Rozing PM. Neonatal screening and staggered early treatment for congenital dislocation or dysplasia of the hip. Lancet. 1990;336:1549-53.

3. Klisic PJ. Congenital dislocation of the hip: a misleading term. Brief report. J Bone Joint Surg. 1989;71:136.

4. Kocher MS. Ultrasonographic screening for developmental dysplasia of the hip: an epidemiologic analysis (part I). Am J Orthop. 2000;29:929-33.

5. Bialik V, Bialik GM. Developmental dysplasia of the hip: a new approach to incidence. Pediatrics. 1999;103:93-9.

6. Beaty JH. Congenital and developmental dysplasia of the hip. In: Canale ST, Beaty JH (eds.). Campbell's Operative Orthopaedics. Philadelphia: Mosby; 2007. p. 1180-98.

7. Hosalkar HS, Horn D, Friedman JE, Dormans JP. The Hip. In: Kliegman RM, Behrman RE, Jenson
$H B$, Stanton BF (eds.). Nelson Textbook of Pediatrics. Philadelphia: Saunders; 2007. p. 2800-5.

8. Harris WH. Etiology of osteoarthritis of the hip. Clin Orthop Relat Res. 1986;213:20-33.

9. Mooney JF, Emans JB. Developmental dislocation of the hip: a clinical overview. Pediatr Rev. 1995;16:299-303.

10. Azzopardi T, Van Essen P. Late diagnosis of developmental dysplasia of the hip: an analysis of risk factors. J Pediatr Orthop B. 2011;20:1-7.

11. Mahan ST, Katz JN, Kim YJ. To screen or not to screen? A decision analysis of the utility of screening for developmental dysplasia of the hip. J Bone Joint Surg Am. 2009;91:1705-19.

12. Lehman HP, Hinton R, Morello P. Developmental dysplasia of the hip practice guideline: technical report. Committee on Quality Improvement, and Subcommittee on Developmental Dysplasia of the Hip. Pediatrics. 2000;105:57.

13. Patel $\mathrm{H}$; Canadian Task Force on Preventive Health Care. Preventive health care, 2001 update: screening and management of developmental dysplasia of the hip in newborns. CMAJ. 2001;164: 1669-77. 
14. Shipman SA, Helfand M, Moyer VA, Yawn $B P$. Screening for developmental dysplasia of the hip: a systematic literature review for the US Preventive Services Task Force. Pediatrics. 2006;117: 557-76.

15. Paton RW, Hossain S, Eccles K. Eight-year prospective targeted ultrasound screening program for instability and at-risk hip joints in developmental dysplasia of the hip. J Pediatr Orthop. 2002;22:338-41.

16. Rabin DL, Barnett CR. Untreated congenital hip disease. A study of the epidemiology, natural history and social aspects of the disease in a Navajo population. Am J Public Health Nations Health. 1965;55:1-44.

17. Weinstein SL. Natural history of congenital hip dislocation and hip dysplasia. Clin Orthop Relat Res. 1987;225:62-76.

18. Gillam SJ, Foss M, Woolaway M. Late presentation of congenital dislocation of the hip: an audit. Br J Gen Pract. 1990;40:236-7.

19. Riboni G, Bellini A, Serantoni S, Rognoni E, Bisanti L. Ultrasound screening for developmental dysplasia of the hip. Pediatr Radiol. 2003;33:47581.

20. Mahan ST, Katz JN, Kim YJ. To screen or not to screen? A decision analysis of the utility of screening for developmental dysplasia of the hip. J Bone Joint Surg Am. 2009;91:1705-19.

21. Walton MJ, Isaacson Z, McMillan D, Hawkes $R$, Atherton WG. The success of management with the Pavlik harness for developmental dysplasia of the hip using a United Kingdom screening programme and ultrasound-guided supervision. J Bone Joint Surg Br. 2010;92:1013-6.
22. Gardner F, Dezateux C, Elbourne D, Gray A, King A, Quinn A. Collaborative Hip Trial Group. The hip trial: psychosocial consequences for mothers of using ultrasound to manage infants with developmental hip dysplasia. Arch Dis Child Fetal Neonatal Ed. 2005;90:17-24.

23. McHale KA, Corbett D. Parental noncompliance with Pavlik harness treatment of infantile hip problems. J Pediatr Orthop. 1989;9:649-52.

24. Treiber M, Tomazic T, Tekauc-Golob A, Zolger J, Korpar B, Burja S et al. Ultrasound screening for developmental dysplasia of the hip in the newborn: a population-based study in the Maribor region, 1997-2005. Wien Klin Wochenschr. 2008; 120:31-6.

25. von Kries $R$, Ihme $N$, Oberle $D$, Lorani $A$, Stark $R$, Altenhofen $L$ et al. Effect of ultrasound screening on the rate of first operative procedures for developmental hip dysplasia in Germany. Lancet. 2003;362:1883-7.

26. Elbourne D, Dezateux C, Arthur R, Clarke NM, Gray A, King A et al. UK Collaborative Hip Trial Group. Ultrasonography in the diagnosis and management of developmental hip dysplasia (UK Hip Trial): clinical and economic results of a multicentre randomised controlled trial. Lancet. 2002; 360:2009-17.

27. Gray A, Elbourne D, Dezateux C, King A, Quinn A, Gardner F. Economic evaluation of ultrasonography in the diagnosis and management of developmental hip dysplasia in the United Kingdom and Ireland. J Bone Joint Surg Am. 2005;87: 2472-9. 